\title{
Application of Amino-Functionalized SBA-15 Type Mesoporous Silica in One-Pot Synthesis of Spirooxindoles
}

\author{
Ghodsi MOHAMMADI ZIARANI ${ }^{1, *}$, Alireza BADIEI ${ }^{2}$, Somayeh MOUSAVI ${ }^{1}$, Negar LASHGARI ${ }^{2}$, \\ Afsaneh SHAHBAZI ${ }^{2}$ \\ ${ }^{1}$ Department of Chemistry, Alzahra University, Vanak Square, P.O. Box 1993893973, Tehran, Iran \\ ${ }^{2}$ School of Chemistry, College of Science, University of Tehran, Tehran, Iran
}

\begin{abstract}
Amino-functionalized SBA-15 (SBA-Pr-NH $\mathrm{N}_{2}$ has been used as a new basic nanocatalyst in the one-pot synthesis of spirooxindole derivatives via the three-component condensation reaction of isatins, activated methylene reagents, and dimedone in an aqueous medium. SBA-Pr- $\mathrm{NH}_{2}$ has been established as an efficient heterogeneous nanoporous solid basic catalyst (pore size of $6 \mathrm{~nm}$ ) that can be easily handled and removed from the reaction mixture by simple filtration, and also recovered and reused without noticeable loss of reactivity.
\end{abstract}

Key words: amino-functionalized SBA-15; spirooxindoles; isatin; nano-reactor; green synthesis

CLC number: $0643 \quad$ Document code: A

Received 15 August 2012. Accepted 11 September 2012.

*Corresponding author.Tel/Fax: +98-2188041344; E-mail: gmziarani@hotmail.com; gmohammadi@alzahra.ac.ir

This work was supported by Alzahra University and university of Tehran.

English edition available online at Elsevier ScienceDirect (http://www.sciencedirect.com/science/journal/18722067).

Spiro compounds composed of cyclic structures fused at a central carbon atom have recently been the subject of considerable attention because of their interesting conformational features and their structural implications on biological systems. Compounds of this structural class represent an important group of naturally occurring substances that are characterized by their extensive biological properties and applications [1]. The spirooxindole ring system is the core structure in a variety of pharmacologically active agents and natural alkaloids [2]. For example, pteropodine and isopteropodine have been shown to modulate the functions of muscarinic receptors in rats [3], whereas spirotryprostatin A and $\mathrm{B}$ have been identified as mammalian cell cycle inhibitors (Scheme 1) [4].

The spirooxindole derivatives have also demonstrated significant potential for use in a wide range of biological applications such as antimicrobial [5], antitumor [6], and therapeutic agents [7]. Of the heterocycles fused to a spirooxindole ring system, functionally substituted $4 \mathrm{H}$-chromenes are of particular utility because they possess important biological activities.

Several methods have been developed for the synthesis of these heterocyclic skeletons. Isatin has been widely used in organic synthesis $[8,9]$, with one example including the preparation of spirooxindole derivatives via a three-component condensation reaction of dimedone with activated methylene reagents in the presence of different catalysts such as $\mathrm{InCl}_{3}$ [10], tetra- $n$-butylammonium fluo- ride (TBAF) [11], $\beta$-cyclodextrin [12], $\mathrm{NH}_{4} \mathrm{Cl}$ [13], triethylbenzylammonium chloride (TEBA) [14], and ethylenediamine diacetate (EDDA) [15].

Mesoporous solids have received considerable attention recently because of their unique features, such as their high specific surface area, large pore volume, controllable and narrowly distributed pore sizes, biocompatibility, and low levels of toxicity. These ordered mesoporous materials (OMMs) have been reported to have potential applications in several areas, including adsorption [16], chromatography [17], drug delivery [18], and catalysis [19]. Of the many
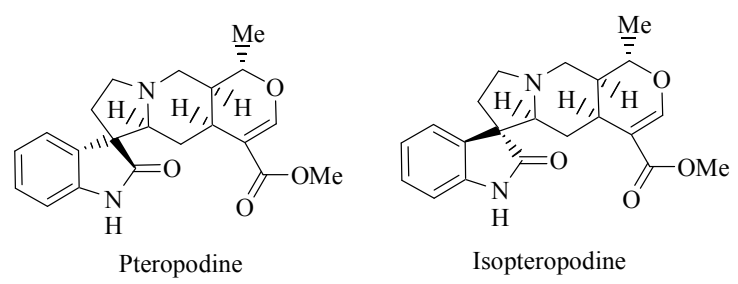

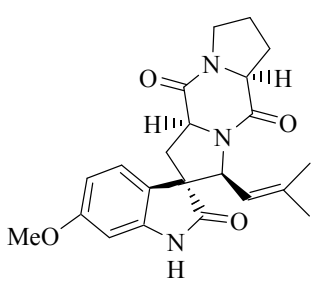

Spirotryprostatin A

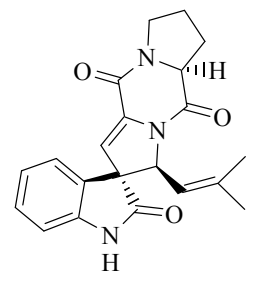

Spirotryprostatin B
Scheme 1. Representative examples of spirooxindole-containing compounds. 
different functionalized silica materials, amino-functionalized SBA-15 is one of the most important functionalized mesoporous materials because it has been used as catalyst in a variety of reactions [20,21]. Considering the biomedical applications of spirooxindole derivatives and in view of the limitations of the existing methods, we were encouraged to use SBA-Pr- $\mathrm{NH}_{2}$ as a catalyst for the facile and efficient multicomponent synthesis of functionalized spirooxindoles.

\section{Experimental}

\subsection{Materials and methods}

All chemicals were obtained commercially and used without further purification. Pluronic P123 $\left(\mathrm{PEO}_{20}-\mathrm{PPO}_{70^{-}}\right.$ $\mathrm{PEO}_{20}, M_{\mathrm{ac}}=5800$ ) block copolymer non-ionic surfactant was purchased from Sigma Aldrich. Tetraethyl orthosilicate (TEOS, 98\%), 3-aminopropyltrimethoxysilane (APTES, $99 \%$ ), hydrochloric acid $(\mathrm{HCl}, 37 \%)$, and toluene were purchased from Merck (Germany). IR spectra were recorded from $\mathrm{KBr}$ disks using an FT-IR Bruker Tensor 27 instrument. Melting points were measured using the capillary tube method with an Electrothermal 9200 apparatus. ${ }^{1} \mathrm{H}$ NMR was run on a Bruker DPX at $500 \mathrm{MHz}$ using TMS as an internal standard. Gas chromatography-mass spectrometry (GC-MS) analysis was performed on an Agilent 6890-5973 GC/MS detector. The low-angle powder X-ray diffraction (XRD) patterns were recorded over a range of $0.5^{\circ}<2 \theta<$ $8^{\circ}$ on a Philips X'pert MPD diffractometer equipped with a liquid nitrogen-cooled germanium solid-state detector using $\mathrm{Cu} K_{\alpha}$ radiation $(40 \mathrm{kV}, 30 \mathrm{~mA})$ at a step width of $0.02^{\circ}$. Nitrogen adsorption-desorption isotherms were measured at $-196{ }^{\circ} \mathrm{C}$ using a BELSORP-mini II. All samples were degassed at $100{ }^{\circ} \mathrm{C}$ for $3 \mathrm{~h}$ under vacuum with an argon gas flow prior to analysis. The specific surface area $\left(A_{\mathrm{BET}}\right)$ was evaluated using the Brunauer-Emmett-Teller (BET) equation, and the pore size distribution $\left(D_{\mathrm{BJH}}\right)$ was obtained from the desorption branches by means of the Barret-Joyner-Halenda (BJH) model, with the pore volume being taken at $p / p_{0}=0.995$. Fourier transform infrared spectra (FT-IR) were recorded within the $600-4000 \mathrm{~cm}^{-1}$ region on a Bruker Vector 22 infrared spectrophotometer to characterize the anchoring functional groups on the silica surface. Transmission electron microscopy (TEM) analysis was performed on a Tecnai $\mathrm{G}^{2} \mathrm{~F} 30$ at $300 \mathrm{kV}$.

\subsection{SBA-15 nanoporous silica synthesis and functionalization}

The synthesis of SBA-15 was similar to that described previously in the literature $[22,23]$ and involved the use of
Pluronic P123 nonionic surfactant as a structure directing agent and TEOS under acidic conditions. Surface modifications over the nanoporous silica with aminopropyl moieties were performed using the post-synthesis grafting method [24]. In a typical process, calcined SBA-15 (5 g) was activated at $200{ }^{\circ} \mathrm{C}$ under vacuum for $5 \mathrm{~h}$ to remove any surface humidity and subsequently refluxed in dry toluene $(150 \mathrm{ml})$. APTES (30.2 mmol) was then slowly added to the mixture and the reaction was refluxed at $110{ }^{\circ} \mathrm{C}$ for a further $24 \mathrm{~h}$. The mixture was then filtered and washed with toluene and any residual organosilane was removed by Soxhlet extraction in ethanol over a $24 \mathrm{~h}$ period. The resulting material was denoted as $\mathrm{NH}_{2}-\mathrm{SBA}-15$.

\subsection{General procedure for the preparation of $\mathrm{N}$-substituted isatins (1e-1g)}

Isatin (10 mmol, $1.47 \mathrm{~g}$ ) or 5-bromoisatin (10 mmol, 2.45 $\mathrm{g})$, potassium carbonate (13 $\mathrm{mmol}, 1.82 \mathrm{~g}$ ), and the corresponding alkyl halide $(11 \mathrm{mmol})$ were dissolved in DMF $(10 \mathrm{ml})$ and heated under reflux at $120{ }^{\circ} \mathrm{C}$. Upon completion of the reaction (monitored by TLC), the crude product was poured in iced water. If the product could be purified by recrystallization, the resulting solid was filtered and washed well with water before being purified by recrystallization from EtOH. If, however, an oily product was formed that resisted crystallization, it was dissolved in ethyl acetate and the organic layer was washed with water and then dried to give the pure product.

\subsection{General procedure for the synthesis of spirooxindole derivatives $(4 a-4 q)$}

The isatin derivative $\mathbf{1 a}-1 \mathbf{g}(2 \mathrm{mmol})$, activated methylene reagent $\mathbf{2 a}-\mathbf{2 c}(2 \mathrm{mmol})$, dimedone $3(2 \mathrm{mmol})$, and SBA-Pr-NH $(0.02 \mathrm{~g})$ were dissolved in water $(5 \mathrm{ml})$ and refluxed (Scheme 2) for the appropriated length of time, as mentioned in Table 3. The progress of the reaction was monitored by TLC. Upon completion of the reaction, the mixture was cooled to room temperature and the crude product filtered off and washed with $\mathrm{H}_{2} \mathrm{O}$. The filter-cake was then collected and dissolved in hot ethanol, and the catalyst was removed by filtration. The filtrates were then cooled to afford pure crystals of the spirooxindole derivatives. The recovered catalyst could then be washed sequentially with diluted aqueous $\mathrm{Et}_{3} \mathrm{~N}$ solution, water, and acetone. Following a period of drying, the catalyst could be reused without any noticeable loss in reactivity.

Methyl-2-amino-5-oxo-7,7-dimethyl-spiro[(4H)-5,6,7,8tetrahydrochromene-4,3'-(3'H)-5'-nitro-indol]-(1'H)-2'-one-3 -carboxylate (4f). IR (KBr, $\left.\mathrm{cm}^{-1}\right): 3379,3189,2954,1727$, $1685,1620,1522,1445,1329,1213,1090,1059,907,746$ 


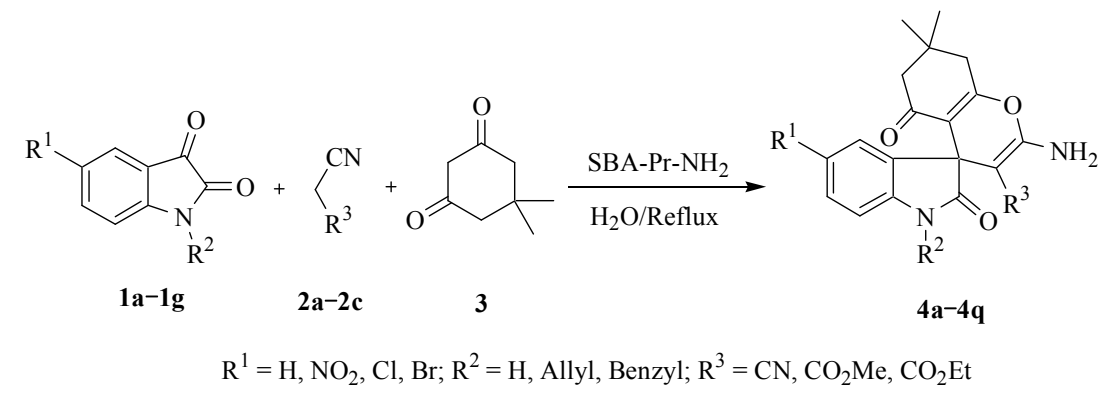

Scheme 2. Synthesis of spirooxindoles $\mathbf{4 a - 4 q}$ in the presence of SBA-Pr-NH

${ }^{1} \mathrm{H}$ NMR (500 MHz, DMSO-d $\left.{ }_{6}\right): \delta 10.95$ (s, 1H, NH), 8.14 (br. s, 2H, $\mathrm{NH}_{2}$ ), 7.74-7.93 (m, 1H, ArH), 6.91 (d, $J=8.0$ $\mathrm{Hz}, 1 \mathrm{H}, \mathrm{ArH}), 6.89$ (d, $J=8.1 \mathrm{~Hz}, 1 \mathrm{H}, \mathrm{ArH}), 3.32$ (s, 3H, $\left.\mathrm{CH}_{3}\right), 2.56\left(\mathrm{~d}, J=15.8 \mathrm{~Hz}, 2 \mathrm{H}, \mathrm{CH}_{2}\right), 2.12(\mathrm{~d}, J=15.9 \mathrm{~Hz}$, $1 \mathrm{H}, \mathrm{CH}_{\mathrm{A}} \mathrm{H}_{\mathrm{B}}$ ), $2.09\left(\mathrm{~d}, J=15.7 \mathrm{~Hz}, 1 \mathrm{H}, \mathrm{CH}_{\mathrm{A}} \mathrm{H}_{\mathrm{B}}\right), 1.00(\mathrm{~s}$, $\left.3 \mathrm{H}, \mathrm{CH}_{3}\right), 0.98$ (s, 3H, $\left.\mathrm{CH}_{3}\right)$. EI-MS: $413\left(\mathrm{M}^{+}\right), 354$ (100), 329.

Methyl-2-amino-5-oxo-7,7-dimethyl-spiro[(4H)-5,6,7,8tetrahydrochromene-4,3'-(3'H)-5'-chloro-indol]-(1'H)-2'-one -3-carboxylate (4g). IR ( $\left.\mathrm{KBr}, \mathrm{cm}^{-1}\right)$ : 3330, 3193, 3134, 2956, 2722, 1733, 1597, 1477, 1303, 1247, 1153, 1051, 963, 780. ${ }^{1} \mathrm{H}$ NMR (500 MHz, DMSO-d 6 ): $\delta 10.28$ (s, 1H, NH), 7.82 (br. s, $2 \mathrm{H}, \mathrm{NH}_{2}$ ), 7.05-7.07 (m, 1H, ArH), 6.87 (d, $J=$ $8.0 \mathrm{~Hz}, 1 \mathrm{H}, \mathrm{ArH}), 6.70$ (d, $J=8.2 \mathrm{~Hz}, 1 \mathrm{H}, \mathrm{ArH}), 3.26$ (s, $\left.3 \mathrm{H}, \mathrm{CH}_{3}\right), 2.52\left(\mathrm{~m}, 2 \mathrm{H}, \mathrm{CH}_{2}\right), 2.14(\mathrm{~d}, J=15.8 \mathrm{~Hz}, 1 \mathrm{H}$, $\left.\mathrm{CH}_{\mathrm{A}} \mathrm{H}_{\mathrm{B}}\right), 2.07\left(\mathrm{~d}, J=15.8 \mathrm{~Hz}, 1 \mathrm{H}, \mathrm{CH}_{\mathrm{A}} \mathrm{H}_{\mathrm{B}}\right), 1.04(\mathrm{~s}, 3 \mathrm{H}$, $\left.\mathrm{CH}_{3}\right), 0.97$ (s, 3H, $\mathrm{CH}_{3}$ ). EI-MS: $402\left(\mathrm{M}^{+}\right), 343$ (100), 318, 304, 260, 83, 55.

Methyl-2-amino-5-oxo-7,7-dimethyl-spiro[(4H)-5,6,7,8tetrahydrochromene-4,3'-(3'H)-5'-bromo-indol]-(1'H)-2'-one -3-carboxylate (4h). IR (KBr, cm $\left.{ }^{-1}\right): 3337,3200,2946$, 1695, 1617, 1522, 1475, 1300, 1224, 1186, 1052, 906, 785. ${ }^{1} \mathrm{H}$ NMR (500 MHz, DMSO-d $\left.)_{6}\right): \delta 10.29$ (s, 1H, NH), 7.85 (br. s, $2 \mathrm{H}, \mathrm{NH}_{2}$ ), 7.21 (d, $\left.J=7.6 \mathrm{~Hz}, 1 \mathrm{H}, \mathrm{ArH}\right), 6.66$ (d, $J=$ $8.0 \mathrm{~Hz}, 1 \mathrm{H}, \mathrm{ArH}), 3.27$ (s, 3H, $\left.\mathrm{CH}_{3}\right), 2.53\left(\mathrm{~m}, 2 \mathrm{H}, \mathrm{CH}_{2}\right)$, $2.15\left(\mathrm{~d}, J=15.8 \mathrm{~Hz}, 1 \mathrm{H}, \mathrm{CH}_{\mathrm{A}} \mathrm{H}_{\mathrm{B}}\right), 2.08(\mathrm{~d}, J=15.8 \mathrm{~Hz}, 1 \mathrm{H}$, $\left.\mathrm{CH}_{\mathrm{A}} \mathrm{H}_{\mathrm{B}}\right), 1.00\left(\mathrm{~s}, 3 \mathrm{H}, \mathrm{CH}_{3}\right), 0.96\left(\mathrm{~s}, 3 \mathrm{H}, \mathrm{CH}_{3}\right)$. EI-MS: 448 $\left(\mathrm{M}^{+}\right), 389$ (100), 362, 309, 281, 250, 83.

Ethyl-2-amino-5-oxo-7,7-dimethyl-spiro[(4H)-5,6,7,8-tetrahydrochromene-4,3'-(3'H)-5'-nitro-indol]-(1'H)-2'-one-3carboxylate $(\mathbf{4 j})$. IR $\left(\mathrm{KBr}, \mathrm{cm}^{-1}\right): 3522,3367,3255,3190$, 2958, 1725, 1686, 1621, 1524, 1470, 1332, 1216, 1170, 1058, 907, 748. ${ }^{1} \mathrm{H}$ NMR (500 MHz, $\left.\mathrm{CDCl}_{3}\right): \delta 10.04(\mathrm{~s}$, $1 \mathrm{H}, \mathrm{NH}), 8.08$ (s, 2H, NH$)_{2}, 6.91-7.71(\mathrm{~m}, 3 \mathrm{H}, \mathrm{ArH}), 3.87$ (q, $\left.J=6.5 \mathrm{~Hz}, 2 \mathrm{H}, \mathrm{CH}_{2}\right), 2.49\left(\mathrm{~m}, 2 \mathrm{H}, \mathrm{CH}_{2}\right), 2.17(\mathrm{~d}, J=$ $\left.16.1 \mathrm{~Hz}, 1 \mathrm{H}, \mathrm{CH}_{\mathrm{A}} \mathrm{H}_{\mathrm{B}}\right), 2.08\left(\mathrm{~d}, J=16.1 \mathrm{~Hz}, 1 \mathrm{H}, \mathrm{CH}_{\mathrm{A}} \mathrm{H}_{\mathrm{B}}\right)$, $1.01\left(\mathrm{~s}, 3 \mathrm{H}, \mathrm{CH}_{3}\right), 0.89$ (s, 3H, $\left.\mathrm{CH}_{3}\right), 0.88(\mathrm{t}, J=7.1 \mathrm{~Hz}, 3 \mathrm{H}$, $\left.\mathrm{CH}_{3}\right)$. EI-MS: $427\left(\mathrm{M}^{+}\right)$, 419, 389, 354, 343, 83, 58.

Ethyl-2-amino-5-oxo-7,7-dimethyl-spiro[(4H)-5,6,7,8-tetrahydrochromene-4,3'-(3'H)-5'-chloro-indol]-(1'H)-2'-one-3 -carboxylate (4k). IR (KBr, $\left.\mathrm{cm}^{-1}\right): 3383,3277,3214,2957$,
1726, 1687, 1614, 1514, 1476, 1350, 1219, 1169, 1052, 907, 747. ${ }^{1} \mathrm{H}$ NMR (500 MHz, DMSO-d ${ }_{6}$ ): $\delta 10.28$ (s, 1H, NH), 7.90 (br. s, 2H, $\mathrm{NH}_{2}$ ), 6.67-7.09 (m, 3H, ArH), 3.72 (q, $J=$ $\left.7.3 \mathrm{~Hz}, 2 \mathrm{H}, \mathrm{CH}_{2}\right), 2.53\left(\mathrm{~m}, 2 \mathrm{H}, \mathrm{CH}_{2}\right), 2.14(\mathrm{~d}, J=15.8 \mathrm{~Hz}$, $\left.1 \mathrm{H}, \mathrm{CH}_{\mathrm{A}} \mathrm{H}_{\mathrm{B}}\right), 2.08\left(\mathrm{~d}, J=15.7 \mathrm{~Hz}, 1 \mathrm{H}, \mathrm{CH}_{\mathrm{A}} \mathrm{H}_{\mathrm{B}}\right), 1.00(\mathrm{~s}$, $\left.3 \mathrm{H}, \mathrm{CH}_{3}\right), 0.96\left(\mathrm{~s}, 3 \mathrm{H}, \mathrm{CH}_{3}\right), 0.81\left(\mathrm{t}, J=7.0 \mathrm{~Hz}, 3 \mathrm{H}, \mathrm{CH}_{3}\right)$. EI-MS: $416\left(\mathrm{M}^{+}\right), 343,153,83,55$.

Ethyl-2-amino-5-oxo-7,7-dimethyl-spiro[(4H)-5,6,7,8-tetrahydrochromene-4,3'-(3'H)-5'-bromo-indol]-(1'H)-2'-one-3 -carboxylate (4I). IR (KBr, cm $\left.{ }^{-1}\right): 3386,3276,3213,2955$, 1726, 1686, 1612, 1512, 1474, 1350, 1216, 1165, 1050, 940, 746. ${ }^{1} \mathrm{H}$ NMR (500 MHz, DMSO-d $\left.{ }_{6}\right): \delta 10.29$ (s, 1H, NH), 7.91 (br. s, 2H, $\mathrm{NH}_{2}$ ), 6.64-7.22 (m, 3H, ArH), 3.72 (q, $J=$ $\left.6.1 \mathrm{~Hz}, 2 \mathrm{H}, \mathrm{CH}_{2}\right), 2.53\left(\mathrm{~m}, 2 \mathrm{H}, \mathrm{CH}_{2}\right), 2.11(\mathrm{~d}, J=15.7 \mathrm{~Hz}$, $\left.1 \mathrm{H}, \mathrm{CH}_{\mathrm{A}} \mathrm{H}_{\mathrm{B}}\right), 2.09\left(\mathrm{~d}, J=15.9 \mathrm{~Hz}, 1 \mathrm{H}, \mathrm{CH}_{\mathrm{A}} \mathrm{H}_{\mathrm{B}}\right), 1.00$ (s, $\left.3 \mathrm{H}, \mathrm{CH}_{3}\right), 0.96\left(\mathrm{~s}, 3 \mathrm{H}, \mathrm{CH}_{3}\right), 0.82\left(\mathrm{t}, J=7.1 \mathrm{~Hz}, 3 \mathrm{H}, \mathrm{CH}_{3}\right)$. EI-MS: $460\left(\mathrm{M}^{+}\right), 444,387$ (100), 378.

Methyl-2-amino-5-oxo-7,7-dimethyl-spiro[(4H)-5,6,7,8tetrahydrochromene-4,3'-(3'H)-1'-benzyl-indol]-(1'H)-2'-one -3-carboxylate (4m). IR $\left(\mathrm{KBr}, \mathrm{cm}^{-1}\right): 3366,2955,1680$, 1610, 1529, 1350, 1311, 1170, 1055, 744. ${ }^{1} \mathrm{H}$ NMR (500 MHz, DMSO-d d $): \delta 7.90$ (s, 1H, ArH), 7.59 (d, $J=7.5 \mathrm{~Hz}$, $2 \mathrm{H}, \mathrm{ArH}), 6.67-7.36\left(\mathrm{~m}, 8 \mathrm{H}, \mathrm{ArH}, \mathrm{NH}_{2}\right), 4.88(\mathrm{~s}, 2 \mathrm{H}$, $\left.\mathrm{NCH}_{2}\right), 3.05\left(\mathrm{~s}, 3 \mathrm{H}, \mathrm{CH}_{3}\right), 2.50-2.62\left(\mathrm{~m}, 2 \mathrm{H}, \mathrm{CH}_{2}\right)$, 2.07-2.19 (m, 2H, $\left.\mathrm{CH}_{2}\right), 1.04\left(\mathrm{~s}, 3 \mathrm{H}, \mathrm{CH}_{3}\right), 0.99$ (s, 3H, $\left.\mathrm{CH}_{3}\right)$. EI-MS: $458\left(\mathrm{M}^{+}\right), 399,367,291,279,91$ (100).

2-Amino-5-oxo-7,7-dimethyl-spiro[(4H)-5,6,7,8-tetrahydrochromene-4,3'-(3'H)-1'-benzyl-5'-bromo-indol]-(1'H)-2'one-3-carbonitrile (4n). IR ( $\left.\mathrm{KBr}, \mathrm{cm}^{-1}\right)$ : 3420, 3321, 3179, $3961,2193,1707,1675,1604,1481,1350,1050,813 .{ }^{1} \mathrm{H}$ NMR (500 MHz, DMSO-d $\left.)_{6}\right): \delta .47(\mathrm{~d}, J=18 \mathrm{~Hz}, 2 \mathrm{H}$, $\mathrm{ArH}), 6.67-7.34$ (m, 8H, ArH, $\left.\mathrm{NH}_{2}\right), 4.92\left(\mathrm{~s}, 2 \mathrm{H}, \mathrm{NCH}_{2}\right)$, 2.58-2.62 (m, 2H, $\left.\mathrm{CH}_{2}\right), 2.06-2.51\left(\mathrm{~m}, 2 \mathrm{H}, \mathrm{CH}_{2}\right), 1.05(\mathrm{~s}$, 6H, $\left.2 \mathrm{CH}_{3}\right)$. EI-MS: $503\left(\mathrm{M}^{+}\right)$, 414, 365, 91 (100), 65, 55.

Ethyl-2-amino-5-oxo-7,7-dimethyl-spiro[(4H)-5,6,7,8-tetrahydrochromene-4,3'-(3'H)-1'-benzyl-5'-Bromo-indol]-(1' H)-2'-one-3-carboxylate (4o). IR $\left(\mathrm{KBr}, \mathrm{cm}^{-1}\right)$ : 3490, 3391, 3279, 2996, 2919, 1679, 1609, 1528, 1310, 1168, 814. ${ }^{1} \mathrm{H}$ NMR (500 MHz, DMSO-d $\left.)_{6}\right): \delta .01(\mathrm{~s}, 1 \mathrm{H}, \mathrm{ArH}), 7.56(\mathrm{~d}, J$ $=7.5 \mathrm{~Hz}, 2 \mathrm{H}, \mathrm{ArH}), 7.12-7.33\left(\mathrm{~m}, 6 \mathrm{H}, \mathrm{ArH}, \mathrm{NH}_{2}\right), 6.64(\mathrm{~d}$, $J=8 \mathrm{~Hz}, 1 \mathrm{H}, \mathrm{ArH}), 4.93\left(\mathrm{~d}, J=16 \mathrm{~Hz}, 1 \mathrm{H}, \mathrm{NCH}_{2}\right), 4.72$ (d, 
$\left.J=15.5 \mathrm{~Hz}, 1 \mathrm{H}, \mathrm{NCH}_{2}\right), 3.79\left(\mathrm{q}, 2 \mathrm{H}, \mathrm{CH}_{2}\right), 2.50-2.57(\mathrm{~m}$, $\left.2 \mathrm{H}, \mathrm{CH}_{2}\right), 2.13\left(\mathrm{~d}, \mathrm{~J}=11 \mathrm{~Hz}, 2 \mathrm{H}, \mathrm{CH}_{2}\right), 1.02$ (s, 3H, $\mathrm{CH}_{3}$ ), 0.98 (s, 3H, $\left.\mathrm{CH}_{3}\right), 0.55$ (t, 3H, $\left.\mathrm{CH}_{3}\right)$. EI-MS: $550\left(\mathrm{M}^{+}\right), 479$, 461, 371, 91 (100), 65.

2-Amino-5-oxo-7,7-dimethyl-spiro[(4H)-5,6,7,8-tetrahydrochromene-4,3'-(3'H)-1'-allyl-indol]-(1'H)-2'-one-3-carbonitrile (4p). IR (KBr, cm $\left.{ }^{-1}\right): 3374,3311,3147,2961,2879$, 2192, 1722, 1658, 1606, 1470, 1349, 1220, 1054, 747. ${ }^{1} \mathrm{H}$ NMR (500 MHz, DMSO-d $\left.)_{6}\right): \delta 7.22\left(\mathrm{~s}, 2 \mathrm{H}, \mathrm{NH}_{2}\right)$, $7.12-7.15(\mathrm{~m}, 1 \mathrm{H}, \mathrm{ArH}), 6.97$ (d, $J=7 \mathrm{~Hz}, 1 \mathrm{H}, \operatorname{ArH})$, 6.91-6.88 (m, 1H, ArH), $6.80(\mathrm{~d}, J=7.5 \mathrm{~Hz}, 1 \mathrm{H}, \mathrm{ArH})$, 5.52-5.61 (m, 1H, $=\mathrm{CH}), 4.85-4.94\left(\mathrm{~m}, 2 \mathrm{H},=\mathrm{CH}_{2}\right)$, 4.34-4.44 (m, 2H, $\left.\mathrm{NCH}_{2}\right), 2.50-2.57\left(\mathrm{~m}, 2 \mathrm{H}, \mathrm{CH}_{2}\right)$, 2.07-2.19 (m, 2H, $\left.\mathrm{CH}_{2}\right), 1.04\left(\mathrm{~s}, 3 \mathrm{H}, \mathrm{CH}_{3}\right), 1.00(\mathrm{~s}, 3 \mathrm{H}$, $\left.\mathrm{CH}_{3}\right)$. EI-MS: $375\left(\mathrm{M}^{+}\right), 335,290,251$ (100), 223, 209, 194, 179, 140, 115, 55.

Ethyl-2-amino-5-oxo-7,7-dimethyl-spiro[(4H)-5,6,7,8-tetrahydrochromene-4,3'-(3'H)-1'-allyl-indol]-(1'H)-2'-one-3 -carboxylate (4q). IR (KBr, cm $\left.{ }^{-1}\right): 3351,3261,3191,2962$, 1688, 1615, 1528, 1350, 1309, 1222, 1052, 744. EI-MS: $422\left(\mathrm{M}^{+}\right), 381,349$ (100), 338, 309, 291, 83, 69, 55.

\section{Results and Discussion}

The XRD pattern of SBA-15 showed the (100), (110), and (200) reflections typical of an ordered mesoscopic structured silica [25] which exhibit a two-dimensional hexagonal symmetrical array of nano-channels (Fig. 1). $\mathrm{NH}_{2}$-SBA-15 was also characterized by the same pattern, indicating that the grafting of APTES did not affect the structural integrity of SBA-15. The porosity and volumetric characteristics of the materials were evaluated by volumetric analyses. In both materials (Fig. 2), the "Type IV" $\mathrm{N}_{2}$ adsorption-desorption isotherms with "H1-type" hysteresis corresponded to condensation and evaporation steps, and were characteristic of periodic mesoporous materials. The textural properties of SBA-15 and $\mathrm{NH}_{2}-\mathrm{SBA}-15$ have been summarized in Table 1. It is noteworthy that the surface

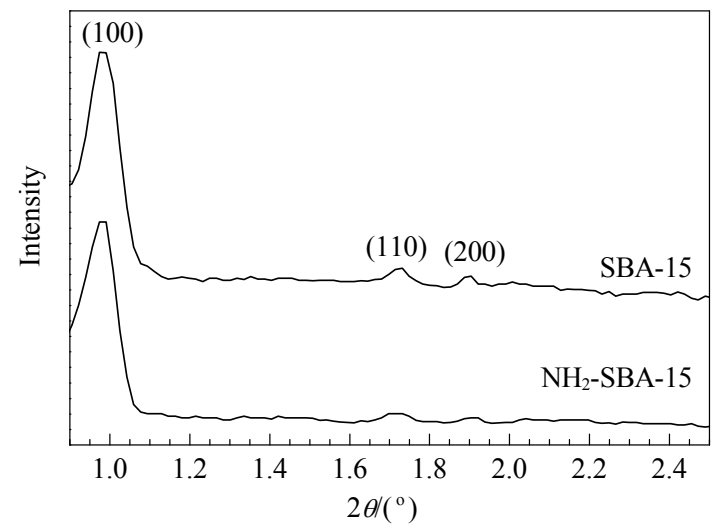

Fig. 1. Low-angle XRD patterns of SBA-15 and $\mathrm{NH}_{2}$-SBA-15.

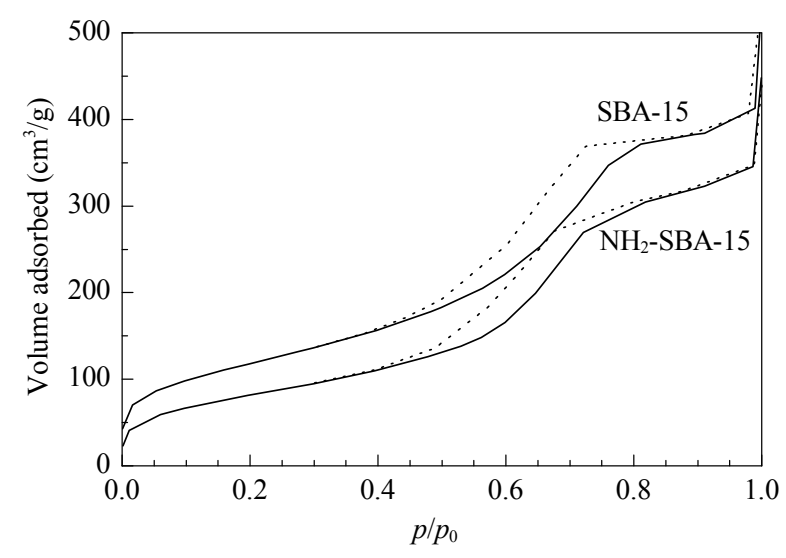

Fig. 2. Nitrogen adsorption-desorption isotherms of SBA-15 and $\mathrm{NH}_{2}$-SBA-15.

area, pore volume, and pore size decreased following the modification, confirming that the surface modification occurred on the inner surface of the silica wall [23].

Table 1 Characteristics of the synthesized materials derived from nitrogen adsorption-desorption

\begin{tabular}{lccc}
\hline Sample & $A_{\mathrm{BET}} /\left(\mathrm{m}^{2} / \mathrm{g}\right)$ & $V_{\text {total }} /\left(\mathrm{cm}^{3} / \mathrm{g}\right)$ & $D_{\mathrm{BJH}} / \mathrm{nm}$ \\
\hline $\mathrm{SBA}-15$ & 481 & 1.3 & 5.9 \\
$\mathrm{NH}_{2}$-SBA-15 & 356 & 1.0 & 3.6 \\
\hline
\end{tabular}

The incorporation of organic groups into the SBA-15 framework was confirmed by the FT-IR spectra (Fig. 3). In both materials, bands around 811 and $1068 \mathrm{~cm}^{-1}$ were seen which were subsequently assigned to the typical symmetric and asymmetric stretching of $\mathrm{Si}-\mathrm{O}-\mathrm{Si}$ caused by the condensed silica network [26]. The broad peak around 3392 $\mathrm{cm}^{-1}$ was assigned to the $\mathrm{O}-\mathrm{H}$ stretching vibration of the $\mathrm{SiO}-\mathrm{H}$ and $\mathrm{HO}-\mathrm{H}$ of adsorbed water. In the spectra of $\mathrm{NH}_{2}$-SBA-15, the bands at 1405 and $1456 \mathrm{~cm}^{-1}$ were assigning to the $-\left(\mathrm{CH}_{2}\right)$ bending vibration, whereas the peaks at 2908 and $2971 \mathrm{~cm}^{-1}$ are attributed to $\mathrm{C}-\mathrm{H}$ stretching vibrations in the methylene groups of the aliphatic chain [22],

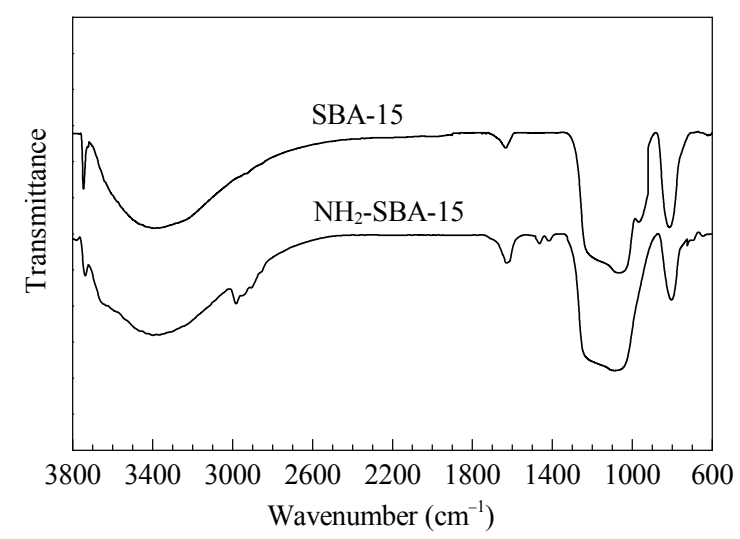

Fig. 3. FT-IR spectra of SBA-15 and $\mathrm{NH}_{2}-\mathrm{SBA}-15$. 
indicating the presence of the anchoring APTES on the silica surface. Moreover, the absorption band at $1548 \mathrm{~cm}^{-1}$, which almost overlapped with the bending vibration of the adsorbed $\mathrm{H}_{2} \mathrm{O}$, corresponded to the $\mathrm{N}-\mathrm{H}$ bending vibration of the $-\mathrm{NH}_{2}$ groups. Therefore, the FT-IR results confirmed the anchoring of the amine groups on the SBA-15 silica surface. The TEM image showed the parallel channels, which resembled the configuration of the pores in SBA-15 (Fig. 4). This indicated that the pores in $\mathrm{NH}_{2}-\mathrm{SBA}-15$ had not collapsed during the functionalization reaction. This image was in good agreement with the XRD result for $\mathrm{NH}_{2}$-SBA-15.

As part of our ongoing interest in the application of heterogeneous solid catalysts in organic synthesis [27-29], herein we report a simple, efficient, and green method for the synthesis of spirooxindole derivatives with fused chromenes, through the three-component condensation of isatin, malononitrile, and dimedone using $\mathrm{SBA}-\mathrm{Pr}^{-} \mathrm{NH}_{2}$ as a nanocatalyst in an aqueous medium.

To establish our optimum conditions, we initially tried to prepare 2-amino-5-oxo-7,7-dimethyl-spiro[(4H)-5,6,7,8tetrahydrochromene-4,3'-(3'H)-5'-bromo-indol]-(1'H)-2'-one -3-carbonitrile $\mathbf{4 d}$ from the reaction of 5-bromo isatin $\mathbf{1}$ (2 $\mathrm{mmol})$, malononitrile 2 ( $2 \mathrm{mmol})$, and dimedone 3 (2 mmol), which we identified as a model reaction, in the presence of SBA-Pr- $\mathrm{NH}_{2}(0.02 \mathrm{~g})$ under different conditions (Table 2). The most encouraging result was obtained when water was employed as the solvent in the presence of a catalytic amount of SBA-Pr- $\mathrm{NH}_{2}$ under refluxing conditions. We decided to investigate the scope of the reaction further by employing a variety of differentially substituted isatins bearing different functionalities and cyanoacetic esters. The

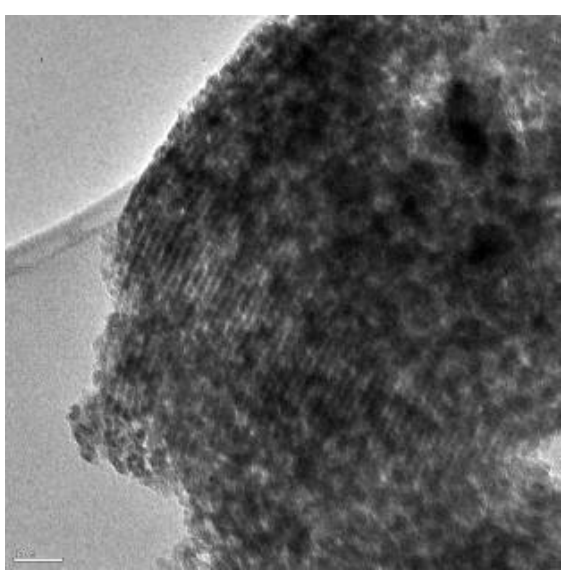

Fig. 4. TEM image of $\mathrm{NH}_{2}-\mathrm{SBA}-15$.

Table 2 The optimization of the reaction conditions for the synthesis of compound 4d

\begin{tabular}{cccc}
\hline Entry & Solvent & Time (min) & Isolated yield (\%) \\
\hline 1 & $\mathrm{H}_{2} \mathrm{O}$ & 5 & 80 \\
2 & $\mathrm{EtOH}$ & 60 & 84 \\
3 & $\mathrm{EtOH} / \mathrm{H}_{2} \mathrm{O}(1: 1)$ & 90 & 73 \\
4 & $\mathrm{CH}_{3} \mathrm{CN}$ & 120 & 75 \\
5 & neat $\left(140{ }^{\circ} \mathrm{C}\right)$ & 180 & 96 \\
\hline
\end{tabular}

corresponding spirooxindole compounds were obtained in high to excellent yields (78-94) after 5-50 min by simple filtration. The condensation reactions of cyanoacetic esters with isatin derivatives and dimedone were also found to give the desired products in high yields (Table 3 ). The progress of the reaction was monitored by TLC. Upon completion of the reaction, the mixture was cooled to room temperature and the crude product was filtered off and washed

Table 3 SBA-Pr- $\mathrm{NH}_{2}$ catalyzed synthesis of spirooxindoles $\mathbf{4 a - 4 q}$ in an aqueous medium

\begin{tabular}{|c|c|c|c|c|c|c|c|c|}
\hline \multirow{2}{*}{ Entry } & \multirow{2}{*}{$\mathrm{R}^{1}$} & \multirow{2}{*}{$\mathrm{R}^{2}$} & \multirow{2}{*}{$\mathrm{R}^{3}$} & \multirow{2}{*}{ Product } & \multirow{2}{*}{$\begin{array}{l}\text { Time } \\
(\mathrm{min})\end{array}$} & \multirow{2}{*}{$\begin{array}{c}\text { Isolated yield } \\
(\%)\end{array}$} & \multicolumn{2}{|c|}{ Melting point $\left({ }^{\circ} \mathrm{C}\right)$} \\
\hline & & & & & & & Found & Reported \\
\hline 1 & $\mathrm{H}$ & $\mathrm{H}$ & $\mathrm{CN}$ & $4 a$ & 5 & 91 & $258-260$ & $268-270[1]$ \\
\hline 2 & $\mathrm{NO}_{2}$ & $\mathrm{H}$ & $\mathrm{CN}$ & $4 b$ & 20 & 94 & $302-304$ & $>300[30]$ \\
\hline 3 & $\mathrm{Cl}$ & $\mathrm{H}$ & $\mathrm{CN}$ & $4 c$ & 5 & 88 & $285-287$ & $286-288[30]$ \\
\hline 4 & $\mathrm{Br}$ & $\mathrm{H}$ & $\mathrm{CN}$ & $4 d$ & 5 & 80 & $302-304$ & $305-307[31]$ \\
\hline 5 & $\mathrm{H}$ & $\mathrm{H}$ & $\mathrm{CO}_{2} \mathrm{Me}$ & $4 e$ & 5 & 79 & $237-239$ & $230-232[32]$ \\
\hline 6 & $\mathrm{NO}_{2}$ & $\mathrm{H}$ & $\mathrm{CO}_{2} \mathrm{Me}$ & $4 f$ & 5 & 85 & $270-271$ & new \\
\hline 7 & $\mathrm{Cl}$ & $\mathrm{H}$ & $\mathrm{CO}_{2} \mathrm{Me}$ & $4 \mathrm{~g}$ & 5 & 80 & $252-254$ & new \\
\hline 8 & $\mathrm{Br}$ & $\mathrm{H}$ & $\mathrm{CO}_{2} \mathrm{Me}$ & $4 h$ & 5 & 79 & $262-264$ & new \\
\hline 9 & $\mathrm{H}$ & $\mathrm{H}$ & $\mathrm{CO}_{2} \mathrm{Et}$ & $4 i$ & 10 & 78 & $260-261$ & $257-258[13]$ \\
\hline 10 & $\mathrm{NO}_{2}$ & $\mathrm{H}$ & $\mathrm{CO}_{2} \mathrm{Et}$ & $4 \mathbf{j}$ & 15 & 81 & $286-289$ & new \\
\hline 11 & $\mathrm{Cl}$ & $\mathrm{H}$ & $\mathrm{CO}_{2} \mathrm{Et}$ & $4 k$ & 25 & 82 & $278-280$ & $271-272[30]$ \\
\hline 12 & $\mathrm{Br}$ & $\mathrm{H}$ & $\mathrm{CO}_{2} \mathrm{Et}$ & 41 & 10 & 83 & $247-248$ & $260-262[33]$ \\
\hline 13 & $\mathrm{H}$ & benzyl & $\mathrm{CO}_{2} \mathrm{Me}$ & $4 m$ & 45 & 88 & $238-240$ & new \\
\hline 14 & $\mathrm{Br}$ & benzyl & $\mathrm{CN}$ & $4 n$ & 30 & 91 & $260-262$ & new \\
\hline 15 & $\mathrm{Br}$ & benzyl & $\mathrm{CO}_{2} \mathrm{Et}$ & 40 & 50 & 85 & $281-283$ & new \\
\hline 16 & $\mathrm{H}$ & allyl & $\mathrm{CN}$ & $4 p$ & 25 & 79 & $>300$ & new \\
\hline 17 & $\mathrm{H}$ & allyl & $\mathrm{CO}_{2} \mathrm{Et}$ & $4 q$ & 35 & 83 & $242-244$ & new \\
\hline
\end{tabular}


with $\mathrm{H}_{2} \mathrm{O}$. The resulting solid was then dissolved in hot ethanol and filtered to remove the catalyst. Subsequent cooling of the filtrate led the precipitation of pure crystals of the spirooxindole derivatives, which were collected by filtration. The recovered catalyst was then washed sequentially with a diluted aqueous $\mathrm{Et}_{3} \mathrm{~N}$ solution, water, and acetone, and dried under vacuum. The reusability of the catalyst was investigated under optimized conditions for the synthesis of the model compound 4a. As shown in Fig. 5, the process of recycling was completed four times with no significant decrease in the activity of the catalyst. The yields for the four runs were found to be $91 \%, 86 \%, 81 \%$, and $72 \%$, respectively.

A possible mechanism for this reaction is shown in Scheme 3. SBA-Pr- $\mathrm{NH}_{2}$ plays a crucial role in accelerating the reaction. The initiation step begins with the deprotonation of malononitrile or the cyanoacetic esters $\mathbf{2}$ by $\mathrm{SBA}-\mathrm{Pr}-\mathrm{NH}_{2}$. It is reasonable to assume that compounds $\mathbf{4 a}-\mathbf{4 q}$ result from the initial formation of the isatylidene malononitrile derivatives 7 according to the standard Knoevenagel reaction. Subsequent Michael-type addition of the enolic form of dimedone $\mathbf{6}$ to $\mathbf{7}$ gives the intermediate 8

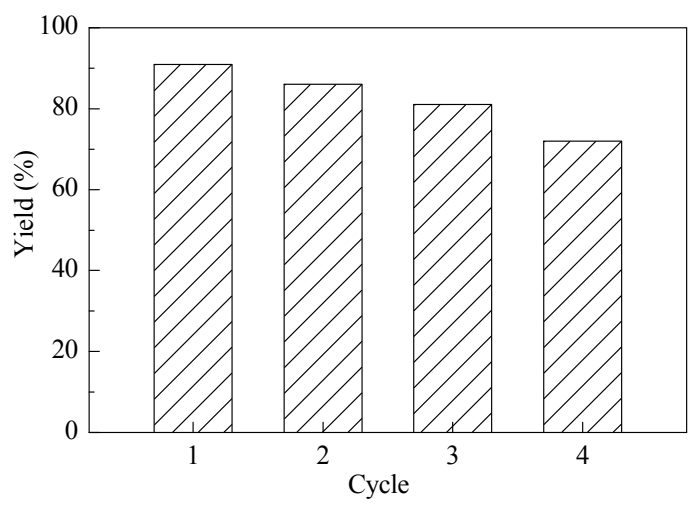

Fig. 5. Reusability of $\mathrm{NH}_{2}-\mathrm{SBA}-15$ in the synthesis of compound $4 \mathbf{a}$.

which affords the corresponding products $\mathbf{4 a - 4 q}$ by cyclization of hydroxyl group to the cyano moiety (Scheme 3 ).

The synthesis of the $N$-alkyl isatins was performed by reacting the isatins with different alkyl halides in DMF in the presence of $\mathrm{K}_{2} \mathrm{CO}_{3}$ for 12-24 $\mathrm{h}$ (Scheme 4) [34]. The results are summarized in Table 4.

Several different conditions have been reported in the literature for the synthesis of spirooxindole derivatives, as

$$
<_{\mathrm{CN}}^{\mathrm{R}^{3}} \stackrel{\mathrm{SBA}-\mathrm{Pr}-\mathrm{NH}_{2}}{\longrightarrow} \Theta<_{\mathrm{CN}}^{\mathrm{R}^{3}}
$$

2
5

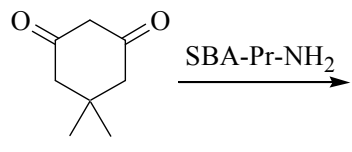

3<smiles>CC1(C)CC(=O)C=C(O)C1</smiles>

6

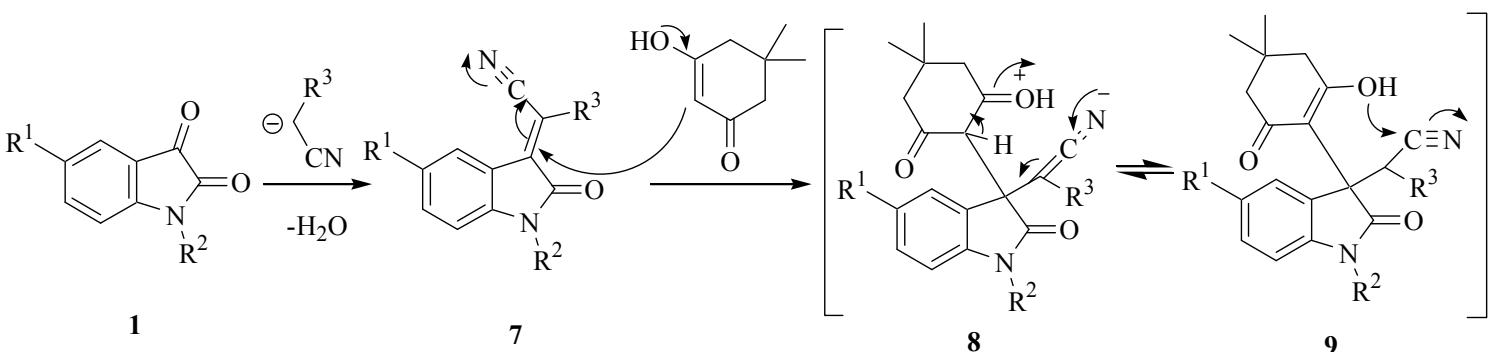

1

7

8

9<smiles>[R]CN=C1[O+]C2=C(C(=O)CC(C)(C)C2)C(C(=O)N([R])c2ccc([Y])cc2)C1[R]</smiles>

10<smiles>[R]C1C(=N)OC2=C(C(=O)CC(C)(C)C2)C1(C)C(=O)N([R])c1ccc(C)cc1</smiles><smiles>[R]C1CC1</smiles><smiles>[R]C(=O)C1(C(=O)N([R])c2ccc([Z13])cc2)C([R])=C(N)OC2=C1C(=O)CC(C)(C)C2</smiles>

11

Scheme 3. Plausible mechanism for synthesis of spirooxindole derivatives $\mathbf{4 a - 4 q}$ in the presence of $\mathrm{SBA}-\mathrm{Pr}-\mathrm{NH}_{2}$.

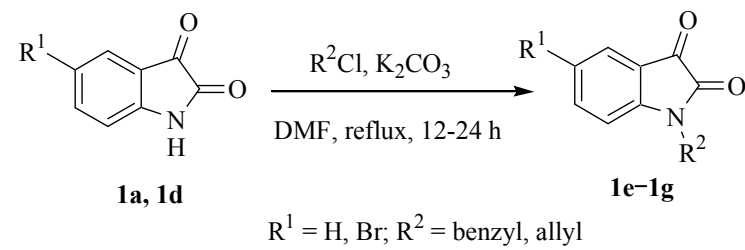

Scheme 4. Synthesis of $N$-alkylated isatins. 
Table 4 Reaction times and yields of $N$-alkylated isatins $1 \mathrm{e}-\mathbf{1 g}$

\begin{tabular}{cccccccc}
\hline \multirow{2}{*}{ Entry } & \multirow{2}{*}{$\mathrm{R}^{1}$} & \multirow{2}{*}{$\mathrm{R}^{2}$} & Product & Time $(\mathrm{h})$ & Yield $(\%)$ & \multicolumn{2}{c}{ Melting point $\left({ }^{\circ} \mathrm{C}\right)$} \\
\cline { 7 - 8 } & & & & & & Found & Reported \\
\hline 1 & $\mathrm{H}$ & benzyl & $\mathbf{1 e}$ & 12 & 85 & $131-133$ & $130-132[35]$ \\
2 & $\mathrm{Br}$ & benzyl & $\mathbf{1 f}$ & 24 & 70 & $147-150$ & $152[36]$ \\
3 & $\mathrm{H}$ & allyl & $\mathbf{1 g}$ & 24 & 80 & $88-91$ & $86-88[37]$ \\
\hline
\end{tabular}

Table 5 Comparison of different conditions for the synthesis of spirooxindole 4a

\begin{tabular}{|c|c|c|c|c|c|c|}
\hline Entry & Catalyst & Solvent & Condition & Time (min) & Yield $(\%)$ & Year \\
\hline 1 & - & $\mathrm{EtOH}$ & electrolysis & 32 & 96 & $2007[31]$ \\
\hline 2 & $\mathrm{InCl}_{3}$ & $\mathrm{CH}_{3} \mathrm{CN}$ & reflux & 90 & 75 & $2007[10]$ \\
\hline 3 & triethylbenzylammonium chloride & $\mathrm{H}_{2} \mathrm{O}$ & heating & 180 & 94 & $2007[14]$ \\
\hline 4 & tris(2-hydroxyethyl)amine & $\mathrm{EtOH}$ & heating & 180 & 95 & $2008[38]$ \\
\hline 5 & tetra- $n$-butylammonium fluoride & $\mathrm{H}_{2} \mathrm{O}$ & reflux & 30 & 97 & $2008[11]$ \\
\hline 6 & $\mathrm{NEt}_{3}$ & $\mathrm{EtOH}$ & reflux & 30 & 83 & $2008[39]$ \\
\hline 7 & $\beta$-Cyclodextrin & $\mathrm{H}_{2} \mathrm{O}$ & heating & 300 & 90 & $2009[12]$ \\
\hline 8 & Ethylenediamine diacetate & $\mathrm{H}_{2} \mathrm{O}$ & heating & 60 & 90 & $2010[15]$ \\
\hline 9 & sodium stearate & $\mathrm{H}_{2} \mathrm{O}$ & heating & 180 & 95 & $2010[40]$ \\
\hline 10 & lipase & $\mathrm{EtOH}$ & heating & 180 & 94 & $2011[30]$ \\
\hline 11 & tetra-n-butylammonium bromide & - & heating & 40 & 90 & $2011[41]$ \\
\hline 12 & SBA-Pr- $\mathrm{NH}_{2}$ & $\mathrm{H}_{2} \mathrm{O}$ & reflux & 5 & 91 & this work \\
\hline
\end{tabular}

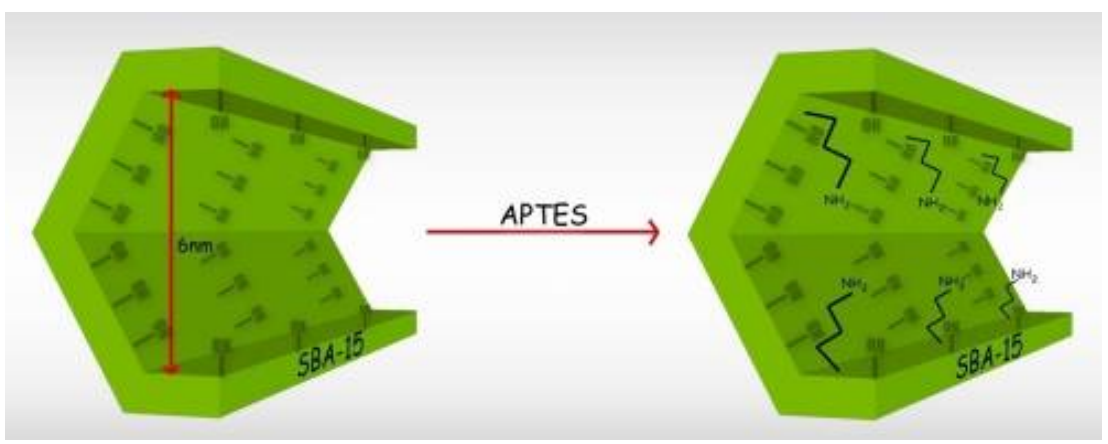

Fig. 7. APTES grafting on the SBA-15 surface.

shown in Table 5. In contrast to the existing methods, the current methodology offers several advantages, such as a simpler procedure, shorter reaction times, facile synthesis, simpler work-up, higher yields, and greener conditions.

The preparation of SBA-15 as a new nanoporous silica can be achieved using the commercially available triblock copolymer Pluronic P123 as a structure directing agent [42]. The amino functionalized SBA-15 was typically synthesized through post-grafting. A schematic illustration of the preparation of SBA-Pr-NH $\mathrm{N}_{2}$ is shown in Fig. 7. The calcined SBA-15 silica was functionalized with APTES to provide SBA-Pr- $\mathrm{NH}_{2}$ as solid basic nanocatalyst.

\section{Conclusions}

We have described an efficient one-pot three-component reaction of isatins with malononitrile or cyanoacetic esters and dimedone for the synthesis of spirooxindole derivatives, catalyzed by SBA-Pr- $\mathrm{NH}_{2}$ in an aqueous medium. The current method does not involve any hazardous organic solvents and the mild reaction conditions and simplicity of the procedure provide significant improvements over many of the other existing methods. We have established SBA-Pr- $\mathrm{NH}_{2}$ as an efficient heterogeneous solid basic catalyst that can be easily handled and removed from the reaction mixture by simple filtration. Short reaction times and high product yields under our reaction conditions clearly demonstrate the advantages of using $\mathrm{SBA}-\mathrm{Pr}-\mathrm{NH}_{2}$ as a nanocatalyst for this reaction.

\section{Acknowledgements}

We gratefully acknowledge for financial support from the Research Councils at Alzahra University and the University of Tehran. 


\section{References}

1 Longeon A, Guyot M, Vacelet J. Experientia, 1990, 46: 548

2 Trost B M, Brennan M K. Synthesis, 2009: 3003

3 Kang TH, Matsumoto K, Tohda M, Murakami Y, Takayama H, Kitajima M, Aimi N, Watanabe H. Eur J Pharmacol, 2002, 444: 39

4 Cui C-B, Kakeya H, Osada H. Tetrahedron, 1996, 52: 12651

5 Bhaskar G, Arun Y, Balachandran C, Saikumar C, Perumal P T. Eur J Med Chem, 2012, 51: 79

6 Jiang X, Sun Y, Yao J, Cao Y, Kai M, He N, Zhang X, Wang Y, Wang R. Adv Synth Catal, 2012, 354: 917

7 Galliford C V, Scheidt K A. Angew Chem, Int Ed, 2007, 46: 8748

8 Da Silva J F M, Garden S J, Pinto A C. J Braz Chem Soc, 2001, 12: 273

9 Lashgari N, Mohammadi Ziarani G. Arkivoc, 2012, i: 277

10 Shanthi G, Subbulakshmi G, Perumal P T. Tetrahedron, 2007, 63: 2057

11 Gao S, Tsai C H, Tseng C, Yao C F. Tetrahedron, 2008, 64: 9143

12 Sridhar R, Srinivas B, Madhav B, Reddy V P, Nageswar Y V D, Rao K R. Can J Chem, 2009, 87: 1704

13 Dabiri M, Bahramnejad M, Baghbanzadeh M. Tetrahedron, 2009, 65: 9443

14 Zhu S-L, Ji S-J, Zhang Y. Tetrahedron, 2007, 63: 9365

15 Hari G S, Lee Y R. Synthesis, 2010: 453

16 Tang T, Zhao Y, Xu Y, Wu D, Xu J, Deng F. Appl Surf Sci, 2011, 257: 6004

17 Yasmin T, Müller K. J Chromatogr A, 2011, 1218: 6464

18 Song S W, Hidajat K, Kawi S. Langmuir, 2005, 21: 9568

19 Ziarani G M, Badiei A R, Khaniania Y, Haddadpour M. Iran J Chem Chem Eng, 2010, 29(2): 1

20 Chen S Y, Huang C Y, Yokoi T, Tang C Y, Huang S J, Lee J J, Chan J C C, Tatsumi T, Cheng S. J Mater Chem, 2012, 22: 2233

21 Sujandi, Prasetyanto E A, Park S E. Appl Catal A, 2008, 350: 244

22 Shahbazi A, Younesi H, Badiei A. Chem Eng J, 2011, 168: 505
23 Badiei A, Goldooz H, Ziarani G M. Appl Surf Sci, 2011, 257: 4912

24 Chong M A S, Zhao X S. J Phys Chem B, 2003, 107: 12650

25 Zhao D, Huo Q, Feng J, Chmelka B F, Stucky G D. J Am Chem Soc, 1998, 120: 6024

26 Badiei A, Goldooz H, Ziarani G M, Abbasi A. J Colloid Interface Sci, 2011, 357: 63

27 Mohammadi Ziarani G, Badiei A, Hassanzadeh M, Mousavi S. Arabian J Chem, 2012, in press

28 Mohammadi Ziarani G, Badiei A, Haddadpour M. Int J Chem, 2011, 3(1): 87

29 Mohammadi Ziarani G, Badiei A, Shahjafari F, Pourjafar T. $S$ Afr J Chem, 2012, 65: 10

30 Chai S-J, Lai Y-F, Xu J-C, Zheng H, Zhu Q, Zhang P-F. Adv Synth Catal, 2011, 353: 371

31 Elinson M N, Ilovaisky A I, Dorofeev A S, Merkulova V M, Stepanov N O, Miloserdov F M, Ogibin Y N, Nikishin G I. Tetrahedron, 2007, 63: 10543

32 Li Y, Chen H, Shi C, Shi D, Ji S. J Comb Chem, 2010, 12: 231

33 Singh Raghuvanshi D, Nand Singh K. J Heterocycl Chem, 2010, 47: 1323

34 Shmidt M S, Reverdito A M, Kremenchuzky L, Perillo I A, Blanco M M. Molecules, 2008, 13: 831

35 Marti C, Carreira E M. J Am Chem Soc, 2005, 127: 11505

36 Majumdar K C, Kundu A K, Chatterjee P. J Chem Res Synop, 1996: 460

37 Ukrainets I V, Bereznyakova N L, Gorokhova O V, Shishkina S V. Chem Heterocycl Compd, 2009, 45: 1241

38 Shemchuk L A, Chernykh V P, Red'kin R G. Russ J Org Chem, 2008, 44: 1789

39 Mortikov V Y, Litvinov Y M, Shestopalov A A, Rodinovskaya L A, Shestopalov A M. Russ Chem Bull Int Ed, 2008, 57: 2373

40 Wang L-M, Jiao N, Qiu J, Yu J-J, Liu J-Q, Guo F-L, Liu Y. Tetrahedron, 2010, 66: 339

41 Mobinikhaledi A, Foroughifar N, Bodaghi Fard M A. Synth Commun, 2011, 41: 441

42 Zhao D, Feng J, Huo Q, Melosh N, Fredrickson G H, Chmelka B F, Stucky G D. Science, 1998, 279: 548 